\title{
Effect of Simultaneous Integrated Boost Intensity Modulated Radiation Therapy (SIB-IMRT) and Non-Operative Strategy on Outcomes of Distal Rectal Cancer Patients with Clinically Positive Lateral Pelvic Lymph Node
}

This article was published in the following Dove Press journal: Cancer Management and Research

\begin{abstract}
Shuai $\mathrm{Li}^{1, *}$
Jianhao Geng ${ }^{l, *}$

Lin Wang $\mathbb{D}^{2, *}$

Huajing Teng $\mathbb{D}^{\prime}$

Zhilong Wang ${ }^{3}$

Xianggao Zhu'

Yangzi Zhang'

Hongzhi Wang'

Yongheng Li (D'

Yong Cai ${ }^{1}$

Aiwen $\mathrm{Wu}^{2}$

Weihu Wang'

'Department of Radiation Oncology, Key Laboratory of Carcinogenesis and Translational Research (Ministry of Education/Beijing), Peking University Cancer Hospital and Institute, Beijing I00 I42, People's Republic of China; ${ }^{2}$ Department of Surgery, Key Laboratory of Carcinogenesis and Translational Research (Ministry of Education), Peking University Cancer Hospital \& Institute, Beijing I00 I42, People's Republic of China; ${ }^{3}$ Department of Radiology, Key Laboratory of

Carcinogenesis and Translational Research (Ministry of Education), Peking University Cancer Hospital \& Institute, Beijing I00 I42, People's Republic of China
\end{abstract}

*These authors contributed equally to this work

Correspondence: Weihu Wang; Aiwen Wu

Tel +86I0-88I96087; +86I0-88I96086

Email wangweihu88@I63.com;

wuaw@sina.com
Background: We aimed to analyze the effect of simultaneous integrated boost intensity modulated radiation therapy (SIB-IMRT) and non-operative treatment on the clinical outcomes of distal rectal cancer patients exhibiting clinically positive lateral pelvic lymph nodes (LPLNs). Methods: We reviewed the medical records of patients diagnosed as having distal rectal adenocarcinoma with clinically positive LPLNs $(\geq 7 \mathrm{~mm}$, with irregular borders or mixed signal intensity) using primary pelvic magnetic resonance imaging (MRI). These patients had received SIB-IMRT-based neoadjuvant chemoradiotherapy (NCRT) and non-operative treatment according to the heterogeneity of the disease or personal preference. Chi-square tests were used to compare data between the two groups. Progression-free survival (PFS) and local regrowth were evaluated using the Kaplan-Meier method.

Results: Between 2016 and 2019, we analyzed 75 patients diagnosed as having clinically positive LPLNs using primary MRI. SIB-IMRT was delivered to the planning positive LPLNs (PGTVn) at a total dose of 56-60 Gy. After NCRT, 23 patients underwent nonoperative treatment. Among these patients, the median short axis of LPLNs was $8 \mathrm{~mm}$ (range: 7-21 mm). Fifteen patients were categorized into the mesorectal fascia (MRF)positive group. The median follow-up duration for these patients was 19.8 months, and no patient exhibited LPLN regrowth. The 2-year PFS rate was $85.6 \%$ for non-operative patients, $74.6 \%$ for operative patients, and $90.0 \%$ for the pathological complete response (pCR) subgroup. Eighteen patients who underwent non-operative treatment were included in the clinical complete response (cCR) subgroup. The 2-year PFS and local regrowth rates in this group were similar to those in patients with clinically negative LPLN who achieved cCR. During NCRT, 21 (28.0\%) patients experienced grade 2-3 acute reversible toxicity.

Conclusions: SIB-IMRT could eliminate metastases in LPLNs in a safe and effective manner, and non-operative strategies may be promising for $\mathrm{cCR}$ patients.

Keywords: rectal cancer, simultaneous integrated boost intensity modulated radiation therapy, lateral pelvic lymph nodes, disease-free survival

\section{Introduction}

For patients with locally advanced rectal cancer (LARC), chemoradiotherapy (CRT) combined with total mesorectal excision (TME) was widely accepted as a standard treatment strategy. ${ }^{1,2}$ Given that preoperative CRT can lead to clinically 
meaningful tumor regression, responses to CRT followed by radical resection may represent a powerful indicator for long-term prognosis. ${ }^{3-5}$ However, the degree of response varied among patients: some showed almost no response, whereas others exhibited a pathological complete response (pCR). Advancements in radiology and endoscopic technology for rectal cancer allow for some patients to be diagnosed with clinical complete response (cCR) prior to surgery by rigorous screening, whose prognosis was consistent with pCR. ${ }^{6}$ Several studies demonstrated the safety and efficacy of non-operative strategies in patients who achieved cCR, which avoided complications associated with radical surgery and allowed for preservation of the anal sphincter. ${ }^{7-10}$ Partial patients may exhibit residual tumors after CRT, although they may opt for non-operative strategies due to personal preference. Investigating outcomes among these patients receiving non-operative treatment may help us to explore the natural course of rectal cancer following chemoradiotherapy.

Lateral pelvic lymph node (LPLN) metastases occurred in $10-25 \%$ of patients with distal rectal cancer and were associated with poor prognosis. ${ }^{11-13}$ In Japan, lateral pelvic lymph node dissection (LPLD) was recommended for patients with advanced distal rectal cancer. ${ }^{14}$ However, in clinical practice, LPLD required longer operation times and excellent surgical skills. Furthermore, during or after LPLD, urinary and male sexual function may be inevitably impaired. ${ }^{15-17}$ One study reported similar rates of local recurrence among patients with low rectal cancer in Japan (extended surgery consisting of LPLD and wider abdominoperineal excision) and the Netherlands (CRT +TME) ${ }^{18}$ However, this finding may not be generalizable to patients with clinically positive LPLNs. Since some studies indicated that the short-axis diameter of LPLNs was associated with long-term prognosis, and standard radiotherapy may not be effective in patients with large LPLNs, which may in turn lead to poor prognosis. ${ }^{19-21}$ Furthermore, some studies reported that decreases in diameter of at least $4 \mathrm{~mm}$ following CRT were associated with low rates of LPLN positivity and local regional recurrence. ${ }^{22,23}$ However, whether these low-risk patients with LPLN positivity after CRT can benefit from nonoperative treatment remains unclear.

LPLN positivity reflects advanced disease, necessitating CRT. Thus, to improve prognosis among patients with advanced LARC, several research groups investigated strategies for strengthening the effect of neoadjuvant radiotherapy or chemotherapy. ${ }^{24-26}$ Simultaneous integrated boost intensity modulated radiation therapy (SIB-IMRT) has been widely applied to increase the dose delivered to the target volume, rather than prolonging the duration of radiotherapy, and research indicated that radiotherapy doseescalation could increase $\mathrm{CR}$ rates among patients with distal rectal cancer. ${ }^{27,28}$ However, to our knowledge, few studies have focused on the effects of SIB-IMRT and nonoperative treatment on clinically positive LPLNs in these patients. In the present retrospective study, we aimed to investigate clinical outcomes among patients treated using this recently developed strategy.

\section{Patients and Methods}

\section{Patient Screening}

From May 2016 to September 2019, we screened patients with pathologically and radiologically confirmed nonmetastatic rectal cancer who had undergone primary MRI and CRT at our center for inclusion in the study. The inclusion criteria were as follows: 1) tumors located within $8 \mathrm{~cm}$ from the anal verge; 2) presence of clinically positive LPLNs; 3) completion of the SIB-IMRT based CRT regimen; 4) Eastern Cooperative Oncology Group performance status (ECOG-PS) ranging from 0 to $1 ; 5$ ) age $\geq 18$ years; 5) good compliance and sufficient follow-up data. Patients with severe cardiovascular disease, cerebrovascular disease, or other types of advanced cancer were excluded. For comparison, we then reviewed the medical records of patients with clinically negative LPLNs who achieved CCR and had undergone non-operative treatment after CRT.

Prior to CRT, we explained the possible benefits and complications of SIB-IMRT, and each patient provided written informed consent for therapy. The treatment protocol was approved by the Ethics Committee of Peking University Cancer Hospital and Institute (No. 2020YJZ71). This study was conducted in accordance with the Declaration of Helsinki.

\section{MRI Evaluation}

Each patient underwent at least two MRI scans (before and after CRT) with either a 1.5-T (GE Healthcare Optima 360, USA) or 3.0-T MRI scanner (GE Healthcare Discovery MR750, USA). For primary MRI, all images were retrospectively reviewed by one experienced radiation oncologist and one experienced gastrointestinal radiologist. The radiation oncologist measured the short-axis diameter, and the radiologists assessed the morphology of LPLNs. If a patient 
undergoes two MRI scans, the MRI simulation is often used as the final measurement basis of the short-axis diameter. The lateral lymph node (LLN) area was delineated in accordance with guidelines for clinical target volume delineation in patients with rectal cancer, ${ }^{29}$ and only the obturator and internal iliac region were included in the analysis (Figure 1). Clinically positive LPLNs were defined as follows: located in the LLN area, with a short-axis diameter $\geq 7 \mathrm{~mm}$ and an irregular border or mixed signal intensity. ${ }^{19,21,30}$ Data regarding mesorectal lymph node metastases, extramural depth of tumor invasion for T3 patients, extramural vascular invasion status (EMVI) and mesorectal fascia (MRF) status were also recorded.

Border and signal intensity were not assessed on postCRT and follow-up MRIs due to difficulties associated with LPLN shrinkage. One gastrointestinal radiologist was responsible for measuring the short axis of the LPLN. The ypT/N stage and tumor regression grade (TRG) were also assessed. One radiation oncologist reviewed the MRI scans during the follow-up to confirm whether the LPLN positivity existence pre-CRT or newly developed.

\section{Preoperative Chemoradiotherapy}

All patients underwent enhanced CT-based simulation using a custom-made belly board and bladder filling in the supine position. The MRI simulation was used as a reference to obtain a more accurate target contour. The primary gross target volume (GTVp) was defined as the primary rectal carcinoma and enlarged lymph node (more than $1 \mathrm{~cm}$ in the short axis) located in the mesorectum, and the clinical target volume (CTV) was delineated based on Valentini's guidelines. ${ }^{29}$ Briefly, the CTV contained the mesorectal region, presacral space, and common iliac and internal iliac lymph node drainage areas, and we introduced GTVn to delineate LPLNs on the basis of CBCT data obtained during radiotherapy at our department. Planning GTVn (PGTVn), planning GTVp (PGTVp), and planning target volume (PTV) were defined

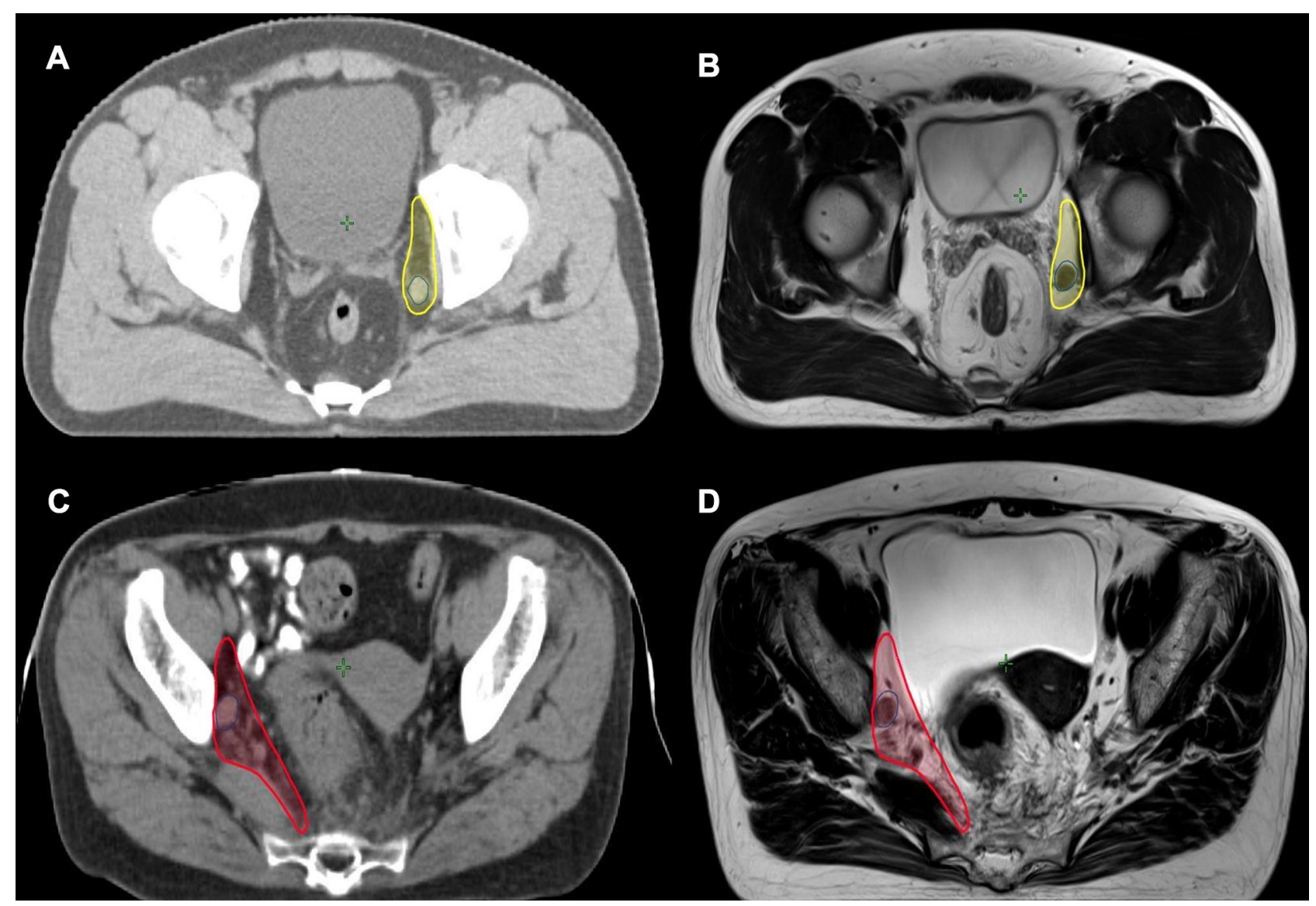

Figure I Two examples of clinically positive LPLNs. (A) Atlas of obturator region (yellow) and the clinically positive LPLN (blue) with 9 mm in short axis; (B) the LPLN and obturator region in MRI simulation; (C) atlas of internal iliac region (red) and the clinically positive LPLN (blue) with II mm in short axis; (D) the LPLN and internal iliac region in MRI simulation. 
as 5-mm extensions of GTVn, GTVp, and CTV in three dimensions, respectively. All patients received intensified radiotherapy by means of SIB-IMRT, and SIB-IMRT was delivered to the PGTVn at 2.54-2.72 Gy per daily fraction, resulting in a total dose of 56-60 Gy. Total radiation doses of 50.6 Gy and 41.8 Gy were delivered to the PGTVp and PTV in 22 daily fractions, respectively.

Two concurrent chemotherapy regimens were administered. The double-agent regimen (CapOX) comprised $85 \mathrm{mg} / \mathrm{m}^{2}$ oxaliplatin every 2 weeks plus $825 \mathrm{mg} / \mathrm{m}^{2}$ capecitabine twice daily for the duration of radiotherapy. Such treatment was recommended for patients with risks such as positive MRF or EMVI. The single-agent group was treated with oral capecitabine $825 \mathrm{mg} / \mathrm{m}^{2}$ twice daily, as described in our previous study. ${ }^{31}$

\section{Induced or Consolidated Chemotherapy}

To improve tumor regression and preserve organ function, total neoadjuvant treatment involving CRT and induced or consolidated chemotherapy may be recommended for patients with partial responses. The chemotherapy regimen depended on patient preference or tumor status, although most opted for the 3-week CapOX- or capecitabine-based regimen.

\section{Evaluations of Toxicity and Outcomes}

During CRT, patients were evaluated weekly by a radiation oncologist, and acute toxicity was graded in accordance with the Common Terminology Criteria of Adverse Events Version 4.0 (CTCAE V4.0). After CRT, additional followup visits and radiological evaluations were planned every 3 months in the first 2 years and every 6 months in the next 3 years.

The primary endpoint was progress-free survival (PFS), which was measured from the date of diagnosis to the date on which any of the following occurred: LPLN regrowth, locoregional recurrence, distant metastases or death. Other endpoints included local regrowth, which was defined as the primary rectal cancer regrowth during the non-operative treatment, and local regrowth was not included in the definition of locoregional recurrence. However, LPLN regrowth or new development of LPLNs in the tumor bed of the LPLD area were defined as recurrence.

\section{Statistical Analyses}

Data were collected and analyzed using the Statistical Package for the Social Sciences (IBM Corp., SPSS
Statistics for Windows, v. 22.0. Armonk, NY, USA), and chi-square tests were used to compare data between the two groups. PFS and local regrowth were evaluated using the Kaplan-Meier method, while the Log rank test was used to compare outcomes among different groups. $P$ values $<0.05$ were considered statistically significant.

\section{Results}

\section{Patient Characteristics}

From May 2016 to September 2019, a total of 75 patients with clinically positive LPLNs received SIB-IMRT-based CRT, and 23 of them underwent non-operative treatment following CRT. Among these 23 patients, the median short-axis diameter of LPLNs was $8 \mathrm{~mm}$ (range: $7-21 \mathrm{~mm}$ ), and the median distance from the tumor to the anal verge was $3 \mathrm{~cm}$ (range: $0-8 \mathrm{~cm}$ ). Fifteen patients were categorized into the MRF-positive group. Among them, nine patients were diagnosed with cT4, six cases of which were due to the tumor invading the levator ani muscle or external anal sphincter. Six patients exhibited clinical positive bilateral LPLNs. Rates of cT4b disease and SIB-IMRT doses were higher in the non-operative group than that in the operative group. Further details for these 75 patients are displayed in Table 1.

In addition to CRT, 15 patients received adjuvant or neoadjuvant chemotherapy for a median of four cycles. Among them, 10 patients underwent the CapOX regimen, four patients underwent treatment with oral capecitabine alone, and one patient underwent combined CapOX and programmed cell death 1 (PD-1) treatment since he was diagnosed with microsatellite instability (MSI) via nextgeneration sequencing (NGS).

\section{Evaluation of LPLNs}

Enhanced pelvic CT (5 $\mathrm{mm}$ thickness) evaluations were performed for the 52 patients who had undergone surgery for primary rectal cancer with or without LPLD. However, 44 patients did not undergo LPLD, and none LPLN involvement was detected on pelvic CT scans after surgery.

MRI evaluations were recommended ( $3 \mathrm{~mm}$ thickness) for the 23 patients in the non-operative group, and the median interval from CRT to the initial MRI evaluation was 2.0 months (range: 0.9-3.5 months). Only three patients exhibited LPLN with diameters $>5 \mathrm{~mm}$ along the short axis, and none of them achieved cCR. However, no LPLN regrowth was noted at the last followup. The second MRI evaluation was performed at 
Table I Clinical Parameters of 75 Patients Who Had Clinically Positive LPLNs

\begin{tabular}{|c|c|c|c|}
\hline Characteristics & $\begin{array}{l}\text { Operative } \\
\text { Group (n = } \\
52 \text { ) }\end{array}$ & $\begin{array}{l}\text { Non-Operative } \\
\text { Group }(n=23)\end{array}$ & P-value \\
\hline Age (range) & $56(30-74)$ & $59(20-83)$ & - \\
\hline $\begin{array}{l}\text { Sex } \\
\qquad \text { Male } \\
\text { Female }\end{array}$ & $\begin{array}{l}36(69.2 \%) \\
16(30.8 \%)\end{array}$ & $\begin{array}{l}17(73.9 \%) \\
6(16.1 \%)\end{array}$ & 0.717 \\
\hline $\begin{array}{l}\text { Pretreatment CEA } \\
\quad \geq 5 \mathrm{~mol} / \mathrm{L} \\
\quad<5 \mathrm{~mol} / \mathrm{L} \\
\text { Unidentified }\end{array}$ & $\begin{array}{l}28(53.8 \%) \\
20(38.5 \%) \\
4(7.7 \%)\end{array}$ & $\begin{array}{l}\text { I3 (56.5\%) } \\
10(43.5 \%) \\
-\end{array}$ & 0.389 \\
\hline $\begin{array}{l}\text { Distance from anal } \\
\text { verge (range) }\end{array}$ & $4(0-8) \mathrm{cm}$ & $3(0-8) \mathrm{cm}$ & - \\
\hline $\begin{array}{l}\text { Clinical T stage } \\
\text { T2 } \\
\text { T3 } \\
\text { T4 }\end{array}$ & $\begin{array}{l}0 \\
44(84.6 \%) \\
8(15.4 \%)\end{array}$ & $\begin{array}{l}3(13.0 \%) \\
\text { II (47.8\%) } \\
9(39.1 \%)\end{array}$ & 0.002 \\
\hline $\begin{array}{l}\text { Clinical N stage } \\
\text { NI } \\
\text { N2 }\end{array}$ & $\begin{array}{l}13(25.0 \%) \\
39(75.0 \%)\end{array}$ & $\begin{array}{l}9(39.1 \%) \\
14(60.9 \%)\end{array}$ & 0.089 \\
\hline $\begin{array}{l}\text { Short axis of LPLN } \\
\text { (range) }\end{array}$ & $8(7-20) \mathrm{mm}$ & $8(7-2 \mathrm{l}) \mathrm{mm}$ & - \\
\hline $\begin{array}{c}\text { MRF status } \\
\text { Positive } \\
\text { Negative }\end{array}$ & $\begin{array}{l}28(53.8 \%) \\
24(46.2 \%)\end{array}$ & $\begin{array}{l}15(65.2 \%) \\
8(34.8 \%)\end{array}$ & 0.359 \\
\hline $\begin{array}{c}\text { EMVI status } \\
\text { Positive } \\
\text { Negative }\end{array}$ & $\begin{array}{l}22(42.3 \%) \\
30(57.7 \%)\end{array}$ & $\begin{array}{l}\text { II (47.8\%) } \\
\text { I2 (52.2\%) }\end{array}$ & 0.657 \\
\hline $\begin{array}{l}\text { Prescribed dose of } \\
\text { LPLN } \\
\quad<60 \mathrm{~Gy} \\
60 \mathrm{~Gy}\end{array}$ & $\begin{array}{l}21(40.4 \%) \\
31(59.6 \%)\end{array}$ & $\begin{array}{l}\text { I ( } 4.3 \%) \\
22(95.7 \%)\end{array}$ & 0.002 \\
\hline $\begin{array}{l}\text { Synchronous } \\
\text { chemotherapy } \\
\text { Capecitabine } \\
\text { CapOX }\end{array}$ & $\begin{array}{l}32(61.5 \%) \\
20(38.5 \%)\end{array}$ & $\begin{array}{l}13(56.5 \%) \\
10(43.5 \%)\end{array}$ & 0.683 \\
\hline $\begin{array}{l}\text { Induced or } \\
\text { consolidated } \\
\text { chemotherapy } \\
\text { Yes } \\
\text { No }\end{array}$ & $\begin{array}{l}27(51.8 \%) \\
25(48.2 \%)\end{array}$ & $\begin{array}{l}\text { I5 (65.2\%) } \\
8(34.8 \%)\end{array}$ & 0.576 \\
\hline
\end{tabular}

Abbreviations: LPLN, lateral pelvic lymph node; CEA, carcinoembryonic antigen; MRF, mesorectal fascia; EMVI, extramural vascular invasion.

a median time of 4.7 months after CRT (range: 3.9-9.7 months), when none of the LPLNs exceeded $5 \mathrm{~mm}$ in the

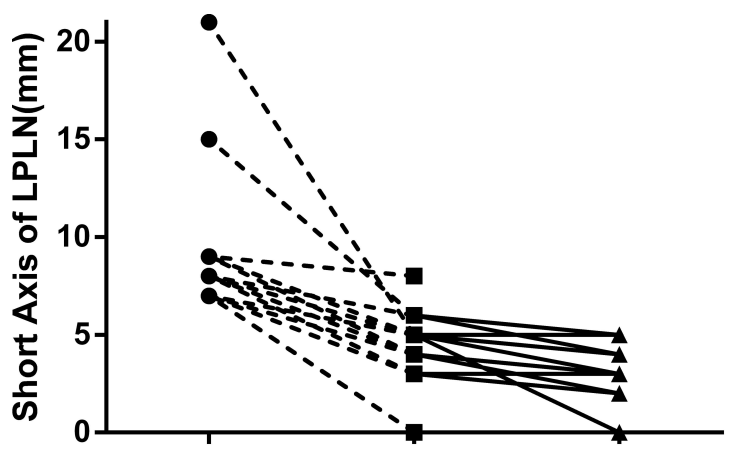

Before CRT First Evaluation Second Evaluation

Figure 2 The changes of short axis for 23 clinically positive LPLN patients who received non-operative strategy.

short axis. The changes in the short-axis diameter of LPLNs are described in Figure 2. No significant changes were observed between the first and second evaluations.

\section{Comparisons Between the Operative and Non-Operative Groups}

The median duration of follow-up for patients with clinically positive LPLNs who had undergone non-operative treatment was 19.8 months (range: 11.5-28.9 months), while that for patients who had undergone operative treatment was 21.2 months (range: 4.7-45.0 months). No LPLN regrowth was observed at the last follow-up.

The 2-year PFS rates for non-operative and operative patients were $85.6 \%$ and $74.6 \%$, respectively $(P=$ $0.147)$. The 2-year PFS rate in the pCR subgroup was $90.0 \%$ (Figure 3). Two patients in the non-operative group (who declined to undergo surgery after CRT) experienced metastasis. Meanwhile, in the surgery group, ten patients experienced metastasis, one patient suffered local recurrence within two years after CRT, and another patient died due to an abdominal infection after surgery.

\section{Toxicity}

During CRT, no patients experienced treatment interruptions or reductions in radiotherapy doses due to toxicity, and no treatment-related deaths occurred. Grade 4 toxicities were not observed in any of the 75 patients, although grade $2-3$ toxicity was noted in $21(28.0 \%)$ patients (Only two patients developed grade 3 toxicity). Only synchronous chemotherapy regimen (CapOX vs single capecitabine: $44.8 \%$ vs $17.4 \%, P=0.010$ ) significantly affected the occurrence of toxicity. Additional details related to 


\section{A}

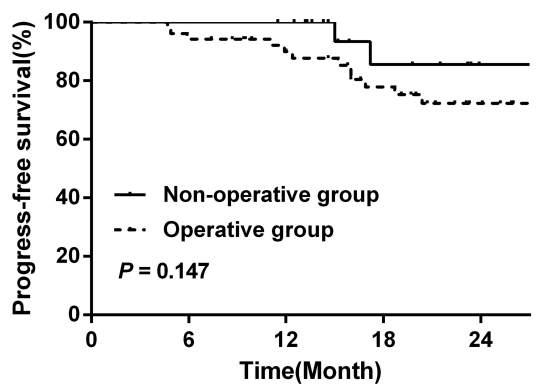

Number at risk: Non-operative group Operative group:

$\begin{array}{ccccc}52 & 49 & 41 & 30 & 10 \\ 23 & 23 & 22 & 11 & 5\end{array}$

B

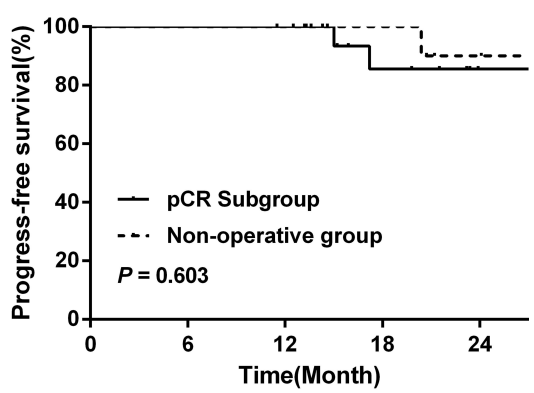

Number at risk: pCR Subgroup: Operative group:

$\begin{array}{lllll}10 & 10 & 10 & 10 & 7 \\ 23 & 23 & 22 & 11 & 5\end{array}$

Figure 3 Comparison of progress-free survival (PFS) of patients with clinically positive LPLN receiving rectal operation or not (A) and comparison between the nonoperative patients and PCR subgroup in operative patients (B).

toxicity are presented in Table 2. Proctitis (17 patients) represented the most common manifestation of toxicity, although this often resolved after CRT. No patients experienced edema of the lower extremities during or after CRT.

Among the patients who underwent surgery after CRT, three $(5.7 \%)$ experienced a small bowel obstruction, while four patients $(7.6 \%)$ experienced infections in the perineal or abdominal wounds. In the non-operative group, one patient who did not achieve cCR declined to undergo surgery and developed a rectovesical fistula 12 months after CRT. During the follow-up period, although patients in the non-operative group often reported tolerable anal pain or changes in bowel habits, none reported regretting selecting this treatment strategy.

\section{Analysis of LPLN Positivity}

During the study period, 48 patients with clinically negative LPLNs achieved cCR after CRT. Eighteen patients in the non-operative group achieved cCR. The clinical parameters are illustrated in Table 3. Rates of MRF positivity $(P=0.000)$, cT4 disease $(P=0.005)$, and abnormal

Table 2 Acute Toxicity in Different Synchronous Chemotherapy Regimens

\begin{tabular}{|l|l|l|l|}
\hline $\begin{array}{l}\text { Toxicity } \\
\text { (Grade 2-3) }\end{array}$ & $\begin{array}{l}\text { CapOX } \\
\text { Regime } \\
\text { (n = 29), } \\
\text { n (\%) }\end{array}$ & $\begin{array}{l}\text { Single Capecitabine } \\
\text { Regime } \\
\text { (n = 46), n (\%) }\end{array}$ & P-value \\
\hline Leukopenia & $3(10.3 \%)$ & $5(10.9 \%)$ & 0.943 \\
Proctitis & $10(34.5 \%)$ & $3(6.5 \%)$ & 0.002 \\
Radiodermatitis & $2(6.9 \%)$ & $2(4.3 \%)$ & 0.632 \\
Anemia & I (3.4\%) & I (2.2\%) & 0.739 \\
Ventosity & I (3.4\%) & 0 & 0.205 \\
\hline
\end{tabular}

carcinoembryonic antigen $(\mathrm{CEA})$ levels $(P=0.006)$ were higher in the clinically positive LPLN group than those in the clinically negative group. These factors were often considered to increase the risk of poor prognosis.

The median duration of follow-up for the clinically positive LPLN group was 18.7 months. The 1- and 2 -year PFS rates were both $100 \%$. For the clinically negative LPLN group, the median duration of follow-up was 22.1 months. The 1- and 2-year PFS rates were 100\% and $96.9 \%$, respectively. No LPLN regrowth was detected during the follow-up period. Local regrowth was observed in seven patients $(14.6 \%)$ from the clinically negative LPLN group. The median time from the end of CRT to local regrowth was 12.8 months (range: 10.6-28.2 months). All seven patients underwent salvage surgery without treatment failure according to the last follow-up. Among them, three patients underwent local excision, two patients underwent low anterior resection, and two patients underwent abdominoperineal resection. Pathological examination revealed that one patient achieved pCR, two experienced TRG 3 based on the National Comprehensive Cancer Network (NCCN) standards, and five exhibited negative PLNs. In the clinically positive LPLD groups, two patients (11.1\%) suffered local regrowth, one patient receive surgery 13.3 months after CRT and pathology, which indicated stage T3N0, and one patient was found to have local regrowth during routine MRI examination 26.6 months after CRT and was admitted for surgery.

\section{Discussion}

To the best of our knowledge, the present study was the first to investigate prognosis among patients undergoing SIB-IMRT and non-operative treatment for distal rectal cancer associated with clinically positive LPLNs. Our 
Table 3 Characteristics of Patients Who Achieved cCR After CRT

\begin{tabular}{|c|c|c|c|}
\hline Characteristics & $\begin{array}{l}\text { Negative } \\
\text { LPLN } \\
\text { Group (n = } \\
48)\end{array}$ & $\begin{array}{l}\text { Clinically } \\
\text { Positive LPLN } \\
\text { Group }(n=18)\end{array}$ & P-value \\
\hline Age (range) & $60(3 I-8 I)$ & $59(36-83)$ & - \\
\hline $\begin{array}{l}\text { Sex } \\
\qquad \text { Male } \\
\text { Female }\end{array}$ & $\begin{array}{l}25(52.1 \%) \\
23(47.9 \%)\end{array}$ & $\begin{array}{l}14 \text { (77.8\%) } \\
4(22.2 \%)\end{array}$ & 0.059 \\
\hline $\begin{array}{l}\text { Pretreatment } \\
\text { CEA } \\
\quad \geq 5 \mathrm{~mol} / \mathrm{L} \\
\quad<5 \mathrm{~mol} / \mathrm{L}\end{array}$ & $\begin{array}{l}8(16.7 \%) \\
40(83.3 \%)\end{array}$ & $\begin{array}{l}9(50.0 \%) \\
9(50.0 \%)\end{array}$ & 0.006 \\
\hline $\begin{array}{l}\text { Distance from } \\
\text { anal verge (range) }\end{array}$ & $3(0-8) \mathrm{cm}$ & $3(0-7) \mathrm{cm}$ & - \\
\hline $\begin{array}{l}\text { Clinical T stage } \\
\text { T2 } \\
\text { T3 } \\
\text { T4 }\end{array}$ & $\begin{array}{l}9(18.8 \%) \\
37(77.1 \%) \\
2(4.2 \%)\end{array}$ & $\begin{array}{l}3(16.7 \%) \\
9(50.0 \%) \\
6(33.3 \%)\end{array}$ & 0.005 \\
\hline $\begin{array}{l}\text { T3 subgroup } \\
\text { T3a } \\
\text { T3b } \\
\text { T3c } \\
\text { T3d }\end{array}$ & $\begin{array}{l}7(14.6 \%) \\
29(60.4 \%) \\
I(2.1 \%) \\
0\end{array}$ & $\begin{array}{l}2(11.1 \%) \\
7(38.9 \%) \\
0 \\
0\end{array}$ & 0.868 \\
\hline $\begin{array}{l}\text { Clinical N stage } \\
\text { N0 } \\
\text { NI } \\
\text { N2 }\end{array}$ & $\begin{array}{l}8(16.7 \%) \\
28(58.3 \%) \\
12(25.0 \%)\end{array}$ & $\begin{array}{l}0 \\
9(50.0 \%) \\
9(50.0 \%)\end{array}$ & 0.057 \\
\hline $\begin{array}{l}\text { MRF status } \\
\text { Positive } \\
\text { Negative }\end{array}$ & $\begin{array}{l}6(12.5 \%) \\
42(87.5 \%)\end{array}$ & $\begin{array}{l}10(55.6 \%) \\
8(44.4 \%)\end{array}$ & 0.000 \\
\hline $\begin{array}{l}\text { EMVI status } \\
\text { Positive } \\
\text { Negative }\end{array}$ & $\begin{array}{l}7(14.6 \%) \\
4 I(85.4 \%)\end{array}$ & $\begin{array}{l}6(33.3 \%) \\
12(66.7 \%)\end{array}$ & 0.088 \\
\hline $\begin{array}{l}\text { Synchronous } \mathrm{CT}^{*} \\
\text { CapOX } \\
\text { Capecitabine }\end{array}$ & $\begin{array}{l}9(18.6 \%) \\
39(81.4 \%)\end{array}$ & $\begin{array}{l}6(33.3 \%) \\
12(66.7 \%)\end{array}$ & 0.208 \\
\hline $\begin{array}{l}\text { Induced or } \\
\text { consolidated CT } \\
\text { Yes } \\
\text { No }\end{array}$ & $\begin{array}{l}33(68.8 \%) \\
15(31.2 \%)\end{array}$ & $\begin{array}{l}12(66.7 \%) \\
6(33.3 \%)\end{array}$ & $0.87 I$ \\
\hline
\end{tabular}

Abbreviations: LPLN, lateral pelvic lymph node; MRF, mesorectal fascia; EMVI, extramural vascular invasion; CT, chemotherapy.

findings indicated that SIB-IMRT was relatively safe, with grade 2-3 acute toxicity occurring in $28 \%$ of patients. Moreover, nearly 2 months after SIB-IMRT, LPLNs had shrunk to a stable status. These findings suggest that nonoperative treatment can lead to similar short-term prognosis as operative treatment in the selected patients, and non-operative strategies maybe feasible for patients with clinical LPLNs positivity who have achieved cCR.

The LPLN was always located beyond the TME boundary and was associated with poor prognosis in patients with distal rectal cancer. ${ }^{1,2,14}$ In one previous study involving 1,977 patients with rectal cancer, 930 underwent LPLD. Positive LPLNs were noted in 129 patients, and survival rates were lower among patients with positive LPLNs than among those with negative LPLNs $(45.8 \%$ vs $71.2 \%, P<0.001){ }^{32}$ However, screening for LPLN positivity remains difficult in real-world clinical settings. A previous retrospective study reported that the incidence of LPLN positivity was higher in patients with low rectal cancer and cT3-4 disease. ${ }^{32}$ In the JCOG0212 study, 351 patients underwent LPLD, and 24 patients exhibited LPLN positivity. Multivariable analysis indicated that tumor locations $(P=0.025)$ and shortaxis diameters $(>5 \mathrm{~mm})$ of the LPLNs $(P=0.002)$ were significantly associated with pathologically positive LPLNs. In our study, rectal tumors were located within $8 \mathrm{~cm}$ of the anal verge in all patients, and most patients exhibited cT3-4 disease. All patients exhibited short-axis diameters greater than $7 \mathrm{~mm}$ so that they had a high probability of LPLN positivity.

NCRT was a standard component of treatment for distal LARC. ${ }^{1,2}$ However, even after NCRT, lateral pelvic recurrence was still among the major causes of locoregional recurrence. Kim et al analyzed data of 366 patients with primary rectal cancer who underwent NCRT and resection, and twenty-nine patients experienced locoregional recurrence, 24 of whom (82.7\%) experienced lateral pelvic recurrence. ${ }^{20}$ Atsushi et al evaluated 1,216 patients with cT3-4 rectal cancers up to $8 \mathrm{~cm}$ from the anal verge. In their study, nearly $80 \%$ of patients had received preoperative radiotherapy, 192 patients had LPLNs with short-axis diameters of at least $7 \mathrm{~mm}$, and multivariate analysis indicated that LPLNs with a shortaxis diameter of at least $7 \mathrm{~mm}$ resulted in significantly higher rates of locoregional recurrence $(\mathrm{HR}=2.060, P=$ $0.045) .{ }^{21}$ Ogura et al conducted a multicenter retrospective study to evaluate risk factors for LPLN positivity based on restaging MRI after NCRT. No cases of lateral recurrence were observed among patients with short-axis diameters of $4 \mathrm{~mm}$ or less, allowing these patients to 
avoid LPLD. ${ }^{33}$ In our study, we utilized SIB-IMRT to strengthen the local effect at LPLNs. In most patients, short-axis diameters had shrunk to $5 \mathrm{~mm}$ by the first radiological evaluation.

The SIB-IMRT technique has been used to improve the down-staging of disease in patients with rectal lesions. Jasna et al launched a Phase 2 study to investigate SIB-IMRT in 51 patients with stage II/III rectal cancer. All patients received IMRT with a pelvic dose of 41.8 Gy in 22 fractions, and SIBIMRT was delivered to T2/3 and T4 tumors at doses of 46.2 Gy and 48.4 Gy, respectively. The authors observed decreased $\mathrm{T}$ stage in $68 \%$ of patients and nodal downstaging in $83 \%$ of patients. The rate of pCR was $25.5 \% .{ }^{34}$ Marco et al assessed 76 patients with LARC who had received pelvic doses of 45 Gy and a median SIB-IMRT dose of 54 Gy (range 52.5-57.5) for tumors. These authors noted that $10.5 \%$ of patients experienced grade $3-4$ acute toxicity, while $20(27.8 \%)$ patients achieved pCR after surgery. In our study, the dose delivered to LPLNs increased to nearly $60 \mathrm{~Gy}$, and no patients exhibited regrowth in the LPLN area. Furthermore, $28.0 \%$ of patients experienced grade $2-3$ acute reversible toxicity. In accordance with previous findings, this result suggests that synchronous chemotherapy regimens can affect toxicity rates. ${ }^{3,35,36}$ In addition, none of our patients exhibited edema in the lower extremities at the last follow-up.

Non-operative strategies have been considered for patients who experienced complete tumor regression. Maas et al evaluated the feasibility and safety of non-operative strategies in 21 patients who achieved cCR in accordance with strict criteria and 20 patients who achieved pCR after surgery. In their study, the 2-year DFS rates were similar in the non-operative and pCR groups (93\% vs $91 \%$ ). The nonoperative group exhibited relatively better functional outcomes in terms of bowel habits. ${ }^{37}$ Among 880 patients who received non-operative treatment, the International Watch \& Wait Database (IWWD) identified 213 patients with local regrowth. Local regrowth occurred in 136 patients $(64 \%)$ in the first year and in 188 patients $(88 \%)$ within 2 years after the decision to undergo non-operative treatment. In the present study, 2-year PFS rates for all patients who underwent non-operative treatment fell between those for the operative group and the pCR subgroup. In addition, two patients (11.1\%) exhibited local regrowth during the last follow-up. Similar to IWWD findings, the median time from the end of CRT to regrowth was 12.8 months in the LPLN-negative group.

The present study had several limitations, including its retrospective design. First, selective bias was inevitable, and before treatment only a few patients underwent NGS and immunohistochemistry for more accurate systemic therapy indications. After NCRT, three patients who underwent surgery at other hospitals were excluded due to an inability to determine the quality of surgery, five patients declined to undergo operation and radiological follow-up, and four patients developed distant metastases. These patients were excluded as well. Second, in the non-operative group, most patients show obvious regression of the tumor and improvement of symptoms; however, because of the COVID-19 pandemic, some patients did not undergo timely radiological evaluation. Lastly, our study was limited by the small sample size and relatively short duration of follow-up, so these results should be generalized with caution.

In conclusion, our findings indicated that SIB-IMRT safely and effectively eliminated suspicious metastases in LPLNs. Furthermore, in selected patients, non-operative strategies led to outcomes similar to those observed in patients negative for LPLN. However, given that local regrowth occurred 10-28 months after NCRT, further studies with longer follow-up periods are required to verify our findings.

\section{Data Sharing Statement}

The datasets used and analyzed during the current study are available from the corresponding author on reasonable request.

\section{Ethics Approval and Consent to Participate}

This retrospective study was approved by the Ethics Committee of Peking University Cancer Hospital and Institute (No. 2020YJZ71).

\section{Funding}

(1) Science Foundation of Peking University Cancer Hospital No. 18-03; (2) Beijing Municipal Science \&Technology Commission No. Z181100001718192; (3) Beijing Natural Science Foundation No. 7182028; (4) Capital's Funds for Health Improvement and Research (2020-2-1027); (5) Sailing Project - Clinical Technology Innovation Project (No. XMLX201842); (6) Capital's Funds for Health Improvement and Research (20202-1027).

\section{Disclosure}

The authors declare that they have no competing interests. 


\section{References}

1. Network. NCC. NCCN Clinical Practice Guidelines in Oncology, Colon Cancer (Version 3.2019). 2019 National Comprehensive Cancer Network Inc. Available from: http://www.ncen.org. Accessed September 26, 2019.

2. Van Cutsem E, Cervantes A, Adam R, et al. ESMO consensus guidelines for the management of patients with metastatic colorectal cancer. Ann Oncol. 2016;27:1386-1422.

3. Aschele C, Cionini L, Lonardi S, et al. Primary tumor response to preoperative chemoradiation with or without oxaliplatin in locally advanced rectal cancer: pathologic results of the STAR-01 randomized Phase III trial. J Clin Oncol. 2011;29:2773-2780.

4. Park IJ, You YN, Agarwal A, et al. Neoadjuvant treatment response as an early response indicator for patients with rectal cancer. $J$ Clin Oncol. 2012;30:1770-1776.

5. Rodel C, Martus P, Papadoupolos T, et al. Prognostic significance of tumor regression after preoperative chemoradiotherapy for rectal cancer. J Clin Oncol. 2005;23:8688-8696.

6. Glynne-Jones R, Wallace M, Livingstone JI, Meyrick-Thomas J. Complete clinical response after preoperative chemoradiation in rectal cancer: is a "wait and see" policy justified? Dis Colon Rectum. 2008;51:10-19; discussion 19-20.

7. Habr-Gama A, Perez RO, Nadalin W, et al. Long-term results of preoperative chemoradiation for distal rectal cancer correlation between final stage and survival. J Gastrointest Surg. 2005;9:90-99.

8. Renehan AG, Malcomson L, Emsley R, et al. Watch-and-wait approach versus surgical resection after chemoradiotherapy for patients with rectal cancer (the OnCoRe project): a propensity-score matched cohort analysis. Lancet Oncol. 2016;17:174-183.

9. Smith JJ, Strombom P, Chow OS, et al. Assessment of a watch-andwait strategy for rectal cancer in patients with a complete response after neoadjuvant therapy. JAMA Oncol. 2019;5:e185896.

10. van der Valk MJM, Hilling DE, Bastiaannet E, et al. Long-term outcomes of clinical complete responders after neoadjuvant treatment for rectal cancer in the International Watch \& Wait Database (IWWD): an international multicentre registry study. Lancet. 2018;391:2537-2545.

11. Hida J, Yasutomi M, Fujimoto K, et al. Does lateral lymph node dissection improve survival in rectal carcinoma? Examination of node metastases by the clearing method. $J$ Am Coll Surg. 1997; 184:475-480.

12. Sauer I, Bacon HE. Influence of lateral spread of cancer of the rectum on radicability of operation and prognosis. Am $J$ Surg. 1951;81:111-120.

13. Yagi R, Shimada Y, Kameyama H, et al. Clinical significance of extramural tumor deposits in the lateral pelvic lymph node area in low rectal cancer: a retrospective study at two institutions. Ann Surg Oncol. 2016;23:552-558.

14. Watanabe T, Itabashi M, Shimada $\mathrm{Y}$, et al. Japanese Society for Cancer of the Colon and Rectum (JSCCR) guidelines 2010 for the treatment of colorectal cancer. Int J Clin Oncol. 2012;17:1-29.

15. Fujita S, Akasu T, Mizusawa J, et al. Postoperative morbidity and mortality after mesorectal excision with and without lateral lymph node dissection for clinical stage II or stage III lower rectal cancer (JCOG0212): results from a multicentre, randomised controlled, non-inferiority trial. Lancet Oncol. 2012;13:616-621.

16. Georgiou P, Tan E, Gouvas N, et al. Extended lymphadenectomy versus conventional surgery for rectal cancer: a meta-analysis. Lancet Oncol. 2009;10:1053-1062.

17. Saito S, Fujita S, Mizusawa J, et al. Male sexual dysfunction after rectal cancer surgery: results of a randomized trial comparing mesorectal excision with and without lateral lymph node dissection for patients with lower rectal cancer: japan Clinical Oncology Group Study JCOG0212. Eur J Surg Oncol. 2016;42:1851-1858.
18. Kusters M, Beets GL, van de Velde CJ, et al. A comparison between the treatment of low rectal cancer in Japan and the Netherlands, focusing on the patterns of local recurrence. Ann Surg. 2009;249:229-235.

19. Shiratori H, Kawai K, Hata K, et al. Correlations between the recurrence patterns and sizes of lateral pelvic lymph nodes before and after chemoradiotherapy in patients with lower rectal cancer. Oncology. 2019;96:33-43.

20. Kim MJ, Kim TH, Kim DY, et al. Can chemoradiation allow for omission of lateral pelvic node dissection for locally advanced rectal cancer? J Surg Oncol. 2015;111:459-464.

21. Ogura A, Konishi T, Cunningham C, et al. Neoadjuvant (Chemo) radiotherapy with total mesorectal excision only is not sufficient to prevent lateral local recurrence in enlarged nodes: results of the multicenter lateral node study of patients with low cT3/4 rectal cancer. J Clin Oncol. 2019;37:33-43.

22. Akiyoshi T, Matsueda K, Hiratsuka M, et al. Indications for lateral pelvic lymph node dissection based on magnetic resonance imaging before and after preoperative chemoradiotherapy in patients with advanced low-rectal cancer. Ann Surg Oncol. 2015;22(Suppl 3):S614-620.

23. Ogura A, Konishi T, Beets GL, et al. Lateral nodal features on restaging magnetic resonance imaging associated with lateral local recurrence in low rectal cancer after neoadjuvant chemoradiotherapy or radiotherapy. JAMA Surg. 2019; 192172.

24. Appelt AL, Ploen J, Vogelius IR, et al. Radiation dose-response model for locally advanced rectal cancer after preoperative chemoradiation therapy. Int J Radiat Oncol Biol Phys. 2013;85:74-80.

25. Deng Y, Chi P, Lan P, et al. Neoadjuvant modified FOLFOX6 with or without radiation versus fluorouracil plus radiation for locally advanced rectal cancer: final results of the Chinese FOWARC trial. J Clin Oncol. 2019;37:3223-3233.

26. Fokas E, Allgauer M, Polat B, et al. Randomized Phase II trial of chemoradiotherapy plus induction or consolidation chemotherapy as total neoadjuvant therapy for locally advanced rectal cancer: $\mathrm{CAO} /$ ARO/AIO-12. J Clin Oncol. 2019;37:3212-3222.

27. Habr-Gama A, Sao Juliao GP, Vailati BB, et al. Organ preservation in cT2N0 rectal cancer after neoadjuvant chemoradiation therapy: the impact of radiation therapy dose-escalation and consolidation chemotherapy. Ann Surg. 2019;269:102-107.

28. Appelt AL, Ploen J, Harling H, et al. High-dose chemoradiotherapy and watchful waiting for distal rectal cancer: a prospective observational study. Lancet Oncol. 2015;16:919-927.

29. Valentini V, Gambacorta MA, Barbaro B, et al. International consensus guidelines on Clinical Target Volume delineation in rectal cancer. Radiother Oncol. 2016;120:195-201.

30. Brown G, Richards CJ, Bourne MW, et al. Morphologic predictors of lymph node status in rectal cancer with use of high-spatial-resolution MR imaging with histopathologic comparison. Radiology. 2003;227:371-377.

31. Li JL, Ji JF, Cai Y, et al. Preoperative concomitant boost intensity-modulated radiotherapy with oral capecitabine in locally advanced mid-low rectal cancer: a phase II trial. Radiother Oncol. 2012;102:4-9.

32. Sugihara K, Kobayashi $\mathrm{H}$, Kato $\mathrm{T}$, et al. Indication and benefit of pelvic sidewall dissection for rectal cancer. Dis Colon Rectum. 2006;49:1663-1672.

33. Ogura A, Konishi T, Beets GL, et al. Lateral nodal features on restaging magnetic resonance imaging associated with lateral local recurrence in low rectal cancer after neoadjuvant chemoradiotherapy or radiotherapy. JAMA Surg. 2019;154:e192172.

34. But-Hadzic J, Anderluh F, Brecelj E, et al. Acute toxicity and tumor response in locally advanced rectal cancer after preoperative chemoradiation therapy with shortening of the overall treatment time using intensity-modulated radiation therapy with simultaneous integrated boost: a Phase 2 trial. Int $J$ Radiat Oncol Biol Phys. 2016;96:1003-1010. 
35. Gerard JP, Azria D, Gourgou-Bourgade S, et al. Comparison of two neoadjuvant chemoradiotherapy regimens for locally advanced rectal cancer: results of the phase III trial ACCORD 12/0405-Prodige 2. J Clin Oncol. 2010;28:1638-1644.

36. O'Connell MJ, Colangelo LH, Beart RW, et al. Capecitabine and oxaliplatin in the preoperative multimodality treatment of rectal cancer: surgical end points from National Surgical Adjuvant Breast and Bowel Project trial R-04. J Clin Oncol. 2014;32:1927-1934.
37. Maas M, Beets-Tan RG, Lambregts DM, et al. Wait-and-see policy for clinical complete responders after chemoradiation for rectal cancer. J Clin Oncol. 2011;29:4633-4640.

\section{Publish your work in this journal}

Cancer Management and Research is an international, peer-reviewed open access journal focusing on cancer research and the optimal use of preventative and integrated treatment interventions to achieve improved outcomes, enhanced survival and quality of life for the cancer patient.
The manuscript management system is completely online and includes a very quick and fair peer-review system, which is all easy to use. Visit http://www.dovepress.com/testimonials.php to read real quotes from published authors. 